Entropy 2009, 11, 111-123; doi:10.3390/e11010111

Article

\title{
Generalized Complexity and Classical-Quantum Transition
}

A. M. Kowalski ${ }^{1,2}$, Angelo Plastino ${ }^{2, \star}$ and Montserrat Casas ${ }^{3}$

${ }^{1}$ Comision de Investigaciones Cientificas (CIC), Argentina

${ }^{2}$ La Plata Physics Institute (IFLP), Exact Sciences Fac., National University (UNLP), Argentina's National Research Council (CCT-CONICET), C.C. 727, (1900) La Plata, Argentina

${ }^{3}$ Departament de Física and IFISC, Universitat de les Illes Balears, 07122 Palma de Mallorca, Spain E-mails: kowalski@ fisica.unlp.edu.ar; montse.casas@uib.es

* Author to whom correspondence should be addressed. E-mail: plastino@ fisica.unlp.edu.ar Received: 10 January 2009/Accepted: 26 February 2009 / Published: 4 March 2009

\begin{abstract}
We investigate the classical limit of the dynamics of a semiclassical system that represents the interaction between matter and a given field. On using as a quantifier the $q$ Complexity, we find that it describes appropriately the quantum-classical transition, detecting the most salient details of the changeover. Additionally the $q$-Complexity results a better quantifier of the problem than the $q$-entropy, in the sense that the $q$-range is enlarged, describing the q-Complexity, the most important characteristics of the transition for all $q$-value.
\end{abstract}

Keywords: Generalized entropy; Semiclassical theories; Quantum chaos; Statistical complexity.

PACS: 89.70.Cf. 03.65.Sq, 05.45.Mt

\section{Introduction}

Quantifiers based on information theory, like entropic forms and statistical complexities (see as examples [1-4]) have proved to be quite useful in the characterization of the dynamics associated to time series, in the wake of the pioneering work of Kolmogorov and Sinai, who converted Shannon's information theory into a powerful tool for the study of dynamical systems [5, 6]. In turn, information theory measures and probability spaces $\Omega$ are inextricably linked quantifiers. In the evaluating them, the determination of the probability distribution $P$ associated to the dynamical system or time series under study is the basic ingredient. Many procedures have been proposed for the election of $P \in \Omega$. We can mention 
techniques based on symbolic dynamics [7], Fourier analysis [8], and wavelet transform [9] (among others). The applicability of these approaches depends on the data-characteristics, i.e., stationarity, length of the series, parameter-variations, levels of noise-contamination, etc. The distinct treatments at hand "capture" the global aspects of the dynamics, but they are not equivalent in their ability to discern physical details. However, one should recognize that we are here referring to techniques defined in an ad-hoc fashion, not derived directly from the dynamical properties of the pertinent system themselves.

Statistical complexity. In [3], López-Ruiz, Mancini and Calbet (LMC) advanced a statistical complexity measure (SCM) based on the notion of "disequilibrium" as a quantifier of the degree of physical structure in a time series. Given a probability distribution associated with a system's state, the LMC measure is the product of an normalized entropy $H$ times a distance to the uniform-equilibrium state $Q$. It vanishes for a totally random process and for a periodic one. Martín et al. [10] improved on this measure by modifying the distance-component (in the concomitant probability space). In Ref. [10], $Q$ is built-up using Wootters' statistical distance while $H$ is a normalized Shannon-entropy. Regrettably enough, the ensuing statistical complexity measure is neither an intensive nor an extensive quantity, although it does yield useful results. A reasonable complexity measure should be able to distinguish among different degrees of periodicity and it should vanish only for periodicity unity. In order to attain such goals it would seem desirable to give this statistical measure an intensive character. This was achieved in Ref. [4] obtaining a SCM that is (i) able to grasp essential details of the dynamics, (ii) an intensive quantity, and (iii) capable of discerning among different degrees of periodicity and chaos.

Deformed $q$-statistics. It is a well-known fact that physical systems that are characterized by either long-range interactions, long-term memories, or multi-fractal nature, are best described by a generalized statistical mechanics' formalism [11] that was proposed 20 years ago: the so-called $q$-statistics. More precisely, Tsallis [12] advanced in 1988 the idea of using in a thermodynamics' scenario an entropic form, the Harvda-Chavrat one, characterized by the entropic index $q \in \mathcal{R}$ ( $q=1$ yields the orthodox Shannon measure):

$$
S_{q}=\frac{1}{(q-1)} \sum_{i=1}^{N_{s}}\left[p_{i}-\left(p_{i}\right)^{q}\right],
$$

where $p_{i}$ are the probabilities associated with the associated $N_{s}$ different system-configurations. The entropic index (or deformation parameter) q describes the deviations of Tsallis entropy from the standard Boltzmann-Gibbs-Shannon-one

$$
S=-\sum_{i=1}^{N_{s}} p_{i} \ln \left(p_{i}\right) .
$$

It is well-known that the orthodox entropy works best in dealing with systems composed of either independent subsystems or interacting via short-range forces whose subsystems can access all the available phase space [11]. For systems exhibiting long-range correlations, memory, or fractal properties, Tsallis' entropy becomes the most appropriate mathematical form [13-16].

Quantum-classical frontier. The classical limit of quantum mechanics (CLQM) continues attracting the attention of many theorists and is the source of much exciting discussion (see, for instance, Refs. [17, 18] and references therein). In particular, the investigation of "quantum" chaotic motion is considered important in this limit. Recent literature provides us with many examples, although the adequate definition of the underlying phenomena is understood in diverse fashion according to the different 
authors (see Ref. [19] and references therein).

It is reasonable to relay on $q$-statistics [36], so as to gather insights into the

$$
\text { quantum - semiclassical - classical transition (CLQM). }
$$

Why? Because we know that the classic to quantum route traverses high complexity regions of the appropriate phase space where chaos reigns, interrupted often by quasi-periodic windows [19-21]. In the semiclassical parcel of the associated trajectory one encounters also strong correlation between classical and quantum degrees of freedom [20,21].

In [36] we showed that a wavelet-evaluated $q$-entropy not only describes correctly the quantumclassical border but also that the associated deformation-parameter $q$ itself characterizes the different regimes involved in the concomitant process, detecting the most salient fine details of the transition. The purpose of the present effort is to gather new insights into the $q$-statistics' contribution to this problem by recourse to a new tool: the $q$-statistical complexity. Since in this work the pertinent $q$-quantifiers are computed using "wavelet techniques" (whose utility has been evidenced in ([20, 21]), we provide a brief wavelet-résumé in the Appendix.

\section{A semi-classical model and the CLQM}

Quite a bit of quantum insight is to be gained from semiclassical perspectives. Several methodologies are available (WKB, Born-Oppenheimer approach, etc.). Here we consider two interacting systems: a classical and a quantal ones. This can be done whenever the quantum effects of one of the two systems are negligible in comparison to those of the other one. Examples can be readily found. We can just mention Bloch-equations [23], two-level systems interacting with an electromagnetic field within a cavity, Jaynes-Cummings semiclassical model [24-27], collective nuclear motion [28], etc. We shall focus attention upon a special bipartite model [29-31] that has been found useful with reference to problems in such diverse fields as chaos, wave-function collapse, measurement processes, and cosmology [32]. In order to investigate the $q$-statistics' contribution to the CLQM problem by recourse to the $q$-statistical complexity (our goal here) we shall consider a trivial generalization of the semi-classical Hamiltonian that represents the zero-th mode contribution of a strong external field to the production of charged meson pairs [30, 31]. It reads

$$
\hat{H}=\frac{1}{2}\left(\frac{\hat{p}^{2}}{m_{q}}+\frac{P_{A}^{2}}{m_{c l}}+m_{q} \omega^{2} \hat{x}^{2}\right),
$$

where $i) \hat{x}$ and $\hat{p}$ are quantum operators, ii) $A$ and $P_{A}$ classical canonical conjugate variables and iii) $\omega^{2}=\omega_{q}^{2}+e^{2} A^{2}$ is an interaction term that introduces nonlinearity, $\omega_{q}$ being a frequency. The quantities $m_{q}$ and $m_{c l}$ are masses, corresponding to the quantum and classical systems, respectively. As shown in Ref. [33], in dealing with (4) one faces an autonomous system of nonlinear coupled equations

$$
\begin{array}{lll}
\frac{d\left\langle\hat{x}^{2}\right\rangle}{d t}=\frac{\langle\hat{L}\rangle}{m_{q}}, & \frac{d\left\langle\hat{p}^{2}\right\rangle}{d t}=-m_{q} \omega^{2}\langle\hat{L}\rangle, & \frac{d\langle\hat{L}\rangle}{d t}=2\left(\frac{\left\langle\hat{p}^{2}\right\rangle}{m_{q}}-m_{q} \omega^{2}\left\langle\hat{x}^{2}\right\rangle\right), \\
\frac{d A}{d t}=\frac{P_{A}}{m_{c l}}, & \frac{d P_{A}}{d t}=-e^{2} m_{q} A\left\langle\hat{x}^{2}\right\rangle, & \hat{L}=\hat{x} \hat{p}+\hat{p} \hat{x} .
\end{array}
$$

The system of Eqs. (5) follows immediately from Ehrenfest's relations [33]. To study the classical limit we need to also consider the classical counterpart of the Hamiltonian (4)

$$
H=\frac{1}{2}\left[\frac{p^{2}}{m_{q}}+\frac{P_{A}^{2}}{m_{c l}}+m_{q}\left(\omega_{q}^{2}+e^{2} A^{2}\right) x^{2}\right],
$$


where all the variables are classical. Recourse to Hamilton's equations allows one to find the classical version of Eqs. (5) (see Ref. [33] for details). Let i) $E$ stand for the total energy of the system and ii) $I$ be an invariant of the motion described by the system (5), related to the Uncertainty Principle, that reads

$$
I=\left\langle\hat{x}^{2}\right\rangle\left\langle\hat{p}^{2}\right\rangle-\frac{\langle\hat{L}\rangle^{2}}{4}
$$

It is easy to see that a classical computation of $I$ yields $I=x^{2} p^{2}-L^{2} / 4 \equiv 0$. The classical limit is obtained by letting [33] the "relative energy"

$$
E_{r}=\frac{|E|}{I^{1 / 2} \omega_{q}} \rightarrow \infty
$$

A measure of the degree of convergence between classical and quantum results in the limit of Eq. (8) is given by the norm $\mathcal{N}$ of the vector $\Delta u=u-u_{c l}$ [33]

$$
\mathcal{N}_{\Delta u}=\left|u-u_{c l}\right|
$$

where the three components vector $u=\left(\left\langle\hat{x}^{2}\right\rangle,\left\langle\hat{p}^{2}\right\rangle,\langle\hat{L}\rangle\right)$ is the "quantum" part of the solution of the system Eqs. (5) and $u_{c l}=\left(x^{2}, p^{2}, L\right)$ its classical partner. A detailed study of our present model was performed in Refs. [33, 34]. We summarize here the main results of these references that are pertinent for our discussion. In plotting diverse dynamical quantities versus $E_{r}$ (as it grows from unity to $\infty$ ), one finds an abrupt change in the system's dynamics for special values of $E_{r}$, to be denoted by $E_{r}{ }^{c l}$. From this value onwards, the pertinent dynamics starts converging to the classical one. It is thus possible to assert that $E_{r}{ }^{c l}$ provides us with an indicator of the presence of a quantum-classical "border". The zone

$$
E_{r}<E_{r}^{c l}
$$

corresponds to the semi-quantal regime investigated in Ref. [34]. This regime, in turn, is characterized by two different sub-zones [33]. i) One of them is an almost purely quantal one, in which the microscopic quantal oscillator is just slightly perturbed by the classical one, and ii) the other section exhibits a transitional nature (semi-quantal). The border between these two sub-zones can be well characterized by a "signal" value $E_{r}{ }^{\mathcal{P}}$. A significant feature of this point resides in the fact that, for $E_{r} \geq E_{r}{ }^{\mathcal{P}}$, chaos is always found. The relative number of chaotic orbits (with respect to the total number of orbits) grows with $E_{r}$ and tends to unity for $E_{r} \rightarrow \infty[33,34]$. Thus, as $E_{r}$ grows from $E_{r}=1$ (the "pure quantum instance") to $E_{r} \rightarrow \infty$ (the classical situation), a significant series of morphology-changes is detected, specially in the transition-zone $\left(E_{r}{ }^{\mathcal{P}} \leq E_{r} \leq E_{r}{ }^{c l}\right)$. The concomitant orbits exhibit features that are not easily describable in terms of Eq. (9), which is a global measure of the degree of convergence in amplitude (of the signal). What one needs instead is a statistical type of characterization, as that described in Refs. [20, 21, 35].

\subsection{Previous q-entropy Results}

In [36], we found that the normalized Tsallis wavelet entropy $\mathcal{H}_{S_{q}}$, in the range $0<q<5$, correctly describes the " $E_{r}$-evolution", identifying the stages of the transition. As a second result we ascertained that within the subrange $0.1<q \leq 0.4, \mathcal{H}_{S_{q}}$, portrays the quantum sector, something that Shannon's 
measure is unable to do, concluding that it is the most appropriate entropy, and not the orthodox, $q=$ 1 of Shannon's. Additionally, we discovered other transition-detectors in addition to the normalized Tsallis-entropy, specially its curvature when we plot it for that particular $q$-value, $q_{M}$, for which $\mathcal{H}_{S_{q}}$ has a minimum. $q_{M}$ itself turned out to a good transition-indicator. These last results affirm that the deformation parameter $q$ by itself can be regarded as the "looking glass" through which one can observe the quantum-classical transition. We are ready now to start presenting the new results of this contribution: the role played by the $q$-complexity in describing the route from the quantum regime to the classical one.

\section{Present results}

\subsection{Introducing the q-statistical complexity}

The Statistical Complexity can be viewed as a functional $C[P]$ that characterizes the probability distribution $P$ associated to the time series generated by the dynamical system under study. It quantifies not only randomness but also the presence of correlational structures $[3,4,10]$. This quantity is of the form [22]

$$
C[P]=Q\left[P, P_{e}\right] H_{S}[P],
$$

where, to the probability distribution $P$, we associate the entropic measure $H_{S}[P]=S[P] / S_{\text {max }}$, with $S_{\text {max }}=S\left[P_{e}\right]\left(0 \leq H_{S} \leq 1\right) . P_{e}$ is the uniform distribution and $S$ is an entropy. We take here the disequilibrium $Q$ to be defined in terms of the extensive Jensen divergence [4] by

$$
Q \equiv Q_{J}\left[P, P_{e}\right]=Q_{0}\left\{S\left[\left(P+P_{e}\right) / 2\right]-S[P] / 2-S\left[P_{e}\right] / 2\right\}
$$

with $Q_{0}$ a normalization constant $\left(0 \leq Q_{J} \leq 1\right)$. We denote the $q-$ entropy (1) by $S$ in (11) and (12). Our wavelet statistical complexity adopts then the following form

$$
\mathcal{C}_{q, J}[P]=Q_{q, J}\left[P, P_{e}\right] \cdot \mathcal{H}_{S_{q}}[P]
$$

with

$$
\mathcal{H}_{S_{q}}[P]=S_{q}[P] / S_{q, \max }=\frac{1}{1-N_{J}^{1-q}} \sum_{j=-1}^{-N_{J}}\left(p_{j}-p_{j}^{q}\right),
$$

a normalized wavelet $q$-entropy (NTWE) (see Appendix) and $Q_{J}\left[P, P_{e}\right]=Q_{0}\left\{S_{q}\left[\left(P+P_{e}\right) / 2\right]-\right.$ $\left.S_{q}[P] / 2-S_{q}\left[P_{e}\right] / 2\right\}$. The set $P \equiv\left\{p_{j}\right\}$ is given by Eq. (19).

\section{Numerical results}

By recourse to the wavelet statistical complexity $\mathcal{C}_{q, J}(13)$, we will be able to characterize the details that pave the road towards the classical limit, accruing additional advantages over the $q$-entropy description. In obtaining our numerical results we choose $m_{q}=m_{c l}=\omega_{q}=e=1$ for the system's parameters. As for the initial conditions for solving the system (5) we take $E=0.6$, i.e., we fix $E$ and then vary $I$ so as to obtain our different $E_{r}$-values. Additionally, we have $\langle L\rangle(0)=L(0)=0$ and $A(0)=0$ (both in the quantum and the classical instances). $\left\langle x^{2}\right\rangle(0)$ takes values in the interval $x^{2}(0)<\left\langle x^{2}\right\rangle(0) \leq 0.502$, with $x^{2}(0)=0.012$. 
Figure 1. $q$-Statistical Complexity $\mathcal{C}_{q, J}$ vs. $E_{r}$ for $q \leq 0.4$ (Fig. 1a) and $0.5 \leq q<1$ (Fig. 1b). Shannon's complexity are also displayed. Three zones are to be differentiated. They are delimited by special $E_{r}$-values, namely, ${E_{r}}^{\mathcal{P}}=3.3282$ and $E_{r}{ }^{c l}=21,55264$. Notice the local complexity maximum at $E_{r}^{M} \simeq 6,8155$.
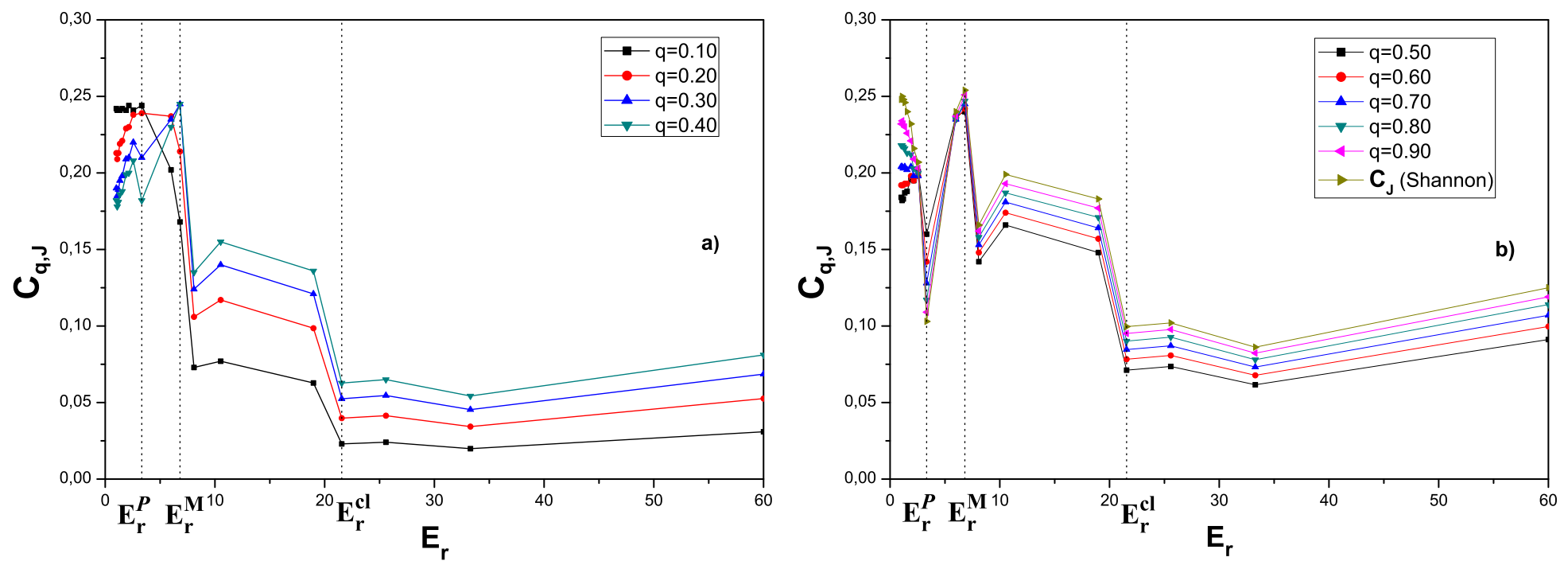

The first task is to evaluate the set $P=\left\{p_{j}\right\}$ with $p_{j}$ given by (19) [Cf. (11) and (13)]. Our data points are the solutions of (5), from which we extract the values of $\left\langle x^{2}\right\rangle$ and the (classical) values of $x^{2}$ at the time $\mathrm{t}$ (for a fixed $E r$ ) (We have also performed these calculations extracting instead $\left\langle p^{2}\right\rangle$ $p^{2}$ together with $\langle L\rangle-L$ and obtained entirely similar results to those reported below). We will deal with $2^{12}$ data-points, for each orbit. We define eight $\left(N_{J}=8\right)$ resolution levels $j=-1,-2, \cdots,-N_{J}$ for an appropriate wavelet analysis within the multi-resolution scheme of the Appendix . The $p_{j}$ yield, at different scales, the energy probability distribution and in very many instances the NTWE has been found to constitute a suitable tool for detecting and characterizing specific phenomena.

We find, as first result, that $\mathcal{C}_{q, J}$ correctly distinguishes the three zones or sections of our process, i.e., quantal, transitional, and classic, as delimited by, respectively, $E_{r}{ }^{\mathcal{P}}=3.3282$ and $E_{r}{ }^{c l}=21,55264$, for all values of $q$, although the quality of the description steadily worsens for $q \rightarrow \infty$ (See Figs. 1, 2, 3, and 4, where we depict $\mathcal{C}_{q, J}$ vs. $E_{r}$ for different q-values). In Fig 1 b) we have include the "Shannon case" $\mathcal{C}_{J}$, i.e., the corresponding wavelet complexity evaluated with the Shannon entropy in (11). Notice the abrupt change of in the slope of the curve taking place at $E_{r}{ }^{\mathcal{P}}$, where a local minimum is detected for $q>0.2$ (Fig 1a). The transition zone is clearly demarcated between that point and $E_{r}{ }^{c l}$. From here on $\mathcal{C}_{q, J}$ tends to its classical value at the same time that the solutions of (5) begin to converge towards the classical ones. There are however some transition-details that are not well represented by $\mathcal{C}_{q, J}$, for some $q$-values. We thus need to ascertain which is the appropriate $q$-range.

In general, the most noticeable $\mathcal{C}_{q, J}$-modifications as $q$ varies take place in the quantal zone, specially for $q<1$ (Figs. 1a-b) and in the transition zone. In the quantum-classic route, an important milestone is found at $E_{r}=E_{r}^{M}$. This point can be detected, within the transition zone, at the value $\approx E_{r}=E_{r}^{M}=$ 6,8155 , for $0.2<q \leq \approx 17$, where a local complexity maximum (lcm) can be appreciated (Figs. 1, 2, 3 and 4) together with a great alteration in the system's dynamics (i.e., in the solutions of (5), which 
Figure 2. $q-$ Statistical Complexity $\mathcal{C}_{q, J}$ vs $E_{r}$ for $1<q<2$. The three zones and the point $E_{r}^{M}$ of Fig. 1 are also seen here.

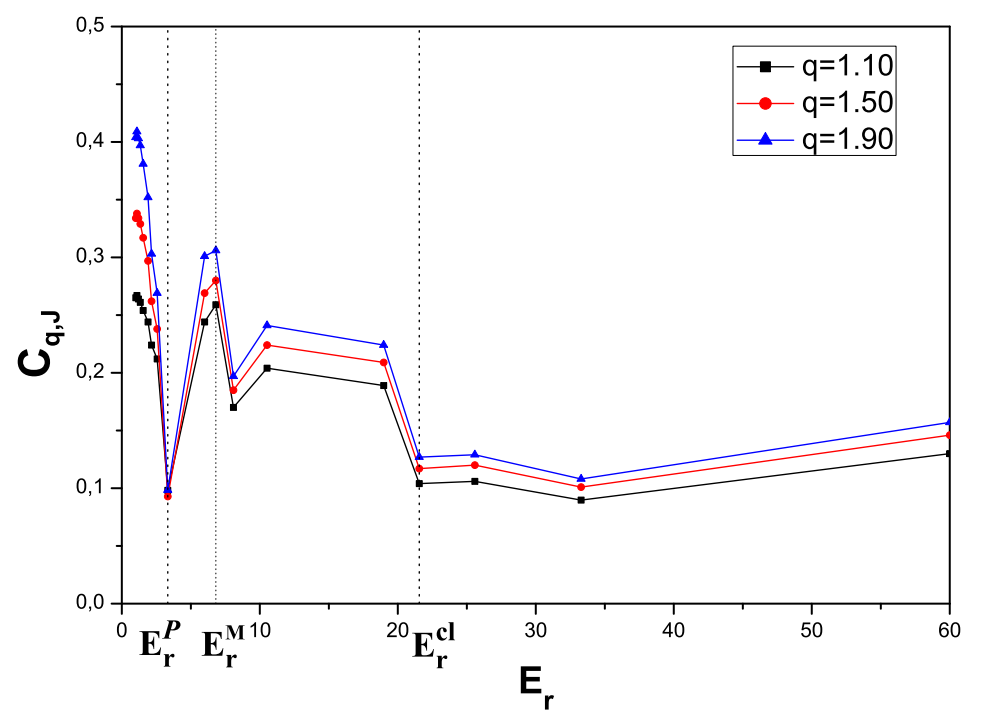

can be verified via Poincare's sections [34]). $E_{r}^{M}$ divides into two sections the transitional region, one in which the quantum-classical mixture characterizes a phase-space with more non-chaotic than chaotic curves and other, in which this feature is reversed [34]. The lcm becomes more pronounced as $q$ grows up to $q=9$, and then becomes less and less noticeable, disappearing for $q>\approx 17$ (Fig. 4).

Notice also that for $0.7 \leq q<1$ (Fig. 1b) and $1<q<2$ (Fig. 2), if $q \rightarrow 1$, the $q$-complexity behavior resembles more and more the Shannon-one of $\mathcal{C}_{J}$. The above picture suffers no great changes for $q \geq 2$, save for the above mentioned changes of the local maximum. In view of these considerations, together with the fact that one obviously wishes for a $\mathcal{H}_{S_{q}}-$ minimum at $E_{r}^{\mathcal{P}}$, we can assert that our $q$-quantifiers should be built up in $q$-range $0.2<q \leq 17$.

The $q$-influence on our transition-processes is clearly appreciated in Figs. 5, that plots $\mathcal{C}_{q, J}$ vs. $q$ for different values of our all important quantity $E_{r}$. The corresponding Shannon statistical complexity value (horizontal line) is included in all graphs for comparison's sake. Figs. 5a)-5b) correspond to the quantum sector, while Figs. 5c), 5d), 5e), and 5f) refer to the transitional one, and, finally, Figs. 5g)-5h) allude to the classical region. Although $\mathcal{H}_{S_{q}}$ possesses only one minimum as a function of $q$ [36], $\mathcal{C}_{q, J}$ instead may exhibit either a minimum and/or a maximum, plus one or more saddle-points. Consequently, $\mathcal{C}_{q, J}$ intersects Shannon's curve $\mathcal{C}_{J}$ at least at one point, i.e., i) at $q=1$ and at one or more points, depending on $E_{r}$.

We find that distinct quantum-zone's graphs resemble each other. Ditto for the classical counterparts. Both kinds are clearly different objects, though. See Figs. 5a)-5b) and 5g)-5h), respectively. Moreover, Fig. 5c displays plots corresponding to the neighborhood of $E_{r}{ }^{\mathcal{P}}$ point, where the transition region begins to exhibit another kind of morphology. For the transition zone two types of picture can be drawn, corresponding to $E_{r} \leq E_{r}^{M}$ (with a phase-space with more non-chaotic than chaotic curves) and $E_{r}>$ $E_{r}^{M}$ (with a phase-space in which this aspect is reversed). The two subregions are displayed in Figs. 5d) - 5e) (corresponding to $E_{r}=E_{r}^{M}$ ) (1st. sub-zone), and Fig 5f) (2nd. subregion). The later tends to resemble the aspect of Figs. $5 \mathrm{~g}$ )-5h) (classical sector). Thus, $\mathcal{C}_{q, J}$ as a function of $q$ is perfectly able, by 
Figure 3. $q-$ Statistical Complexity $\mathcal{C}_{q, J}$ vs. $E_{r}$ for $2 \leq q \leq 5$. No great changes are observed.

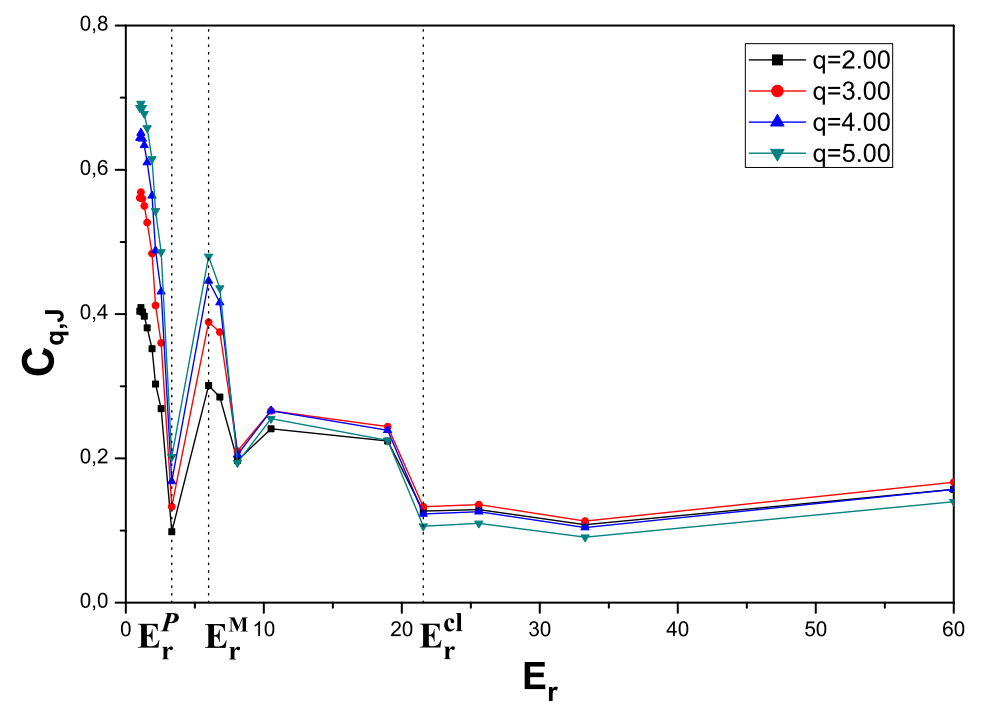

itself, i) of "detecting" important dynamical features like the "Signal Point" $E_{r}{ }^{\mathcal{P}}$ and ii) of distinguishing between the two transitional sub-regions, and iii) registering the similarities between the second of these two and the classical one.

\section{Conclusions}

We have studied in this communication, the classical-quantal frontier of the dynamics governed by a semi-classical Hamiltonian that represents the zero-th mode contribution of an strong external field to the production of charged meson pairs. This study was encompassed within the strictures of the so-called $q$-statistics and by recourse to a new tool: the $q$-Statistical Complexity (13) evaluated by performing a wavelet-band analysis.

The highlights of the road towards classicality are described by recourse to the relative energy $E_{r}$ given by (8). As $E_{r}$ grows from $E_{r}=1$ (the "pure quantum instance") to $E_{r} \rightarrow \infty$ (the classical situation), a significant series of morphology-changes is detected for the solutions of the system of nonlinear coupled equations (5). The concomitant process takes place in three stages: quantal, transitional, and classic, delimited, respectively, by special values of $E_{r}$, namely, $E_{r}{ }^{\mathcal{P}}$ and $E_{r}{ }^{c l}$.

We encounter as a first result that $\mathcal{C}_{q, J}$ distinguishes correctly for all value of $q$, the three sections of our process, i.e., quantal, transitional, and classic, as delimited by, respectively, $E_{r}{ }^{\mathcal{P}}$ and $E_{r}{ }^{c l}$. The description suffers a gradual deterioration process as $q \rightarrow \infty$, a rather important result in view of the fact that the $q$-entropy $\mathcal{H}_{S_{q}}$ is only able to distinguish our three regions in the range $0<q<5$. Such a fact makes the $q$-Statistical Complexity a much better quantifier than the $q$-Entropy for the description of a very involved process. As a second we determine an optimal $q-$ range $\mathcal{O}=[0.2<q \leq 17]$, much larger than the above quoted one for $\mathcal{H}_{S_{q}}$. Within $\mathcal{O}$ our complexity-tool distinguishes a value $E_{r}=E_{r}^{M}$ within the transition zone (TZ) in which the complexity exhibits a local maximum. We can partition the TZ at $E_{r}=E_{r}^{M}$ into two subsections: one in which the quantum-classical mixture characterizes a phase-space with more non-chaotic than chaotic curves and another in which this aspect is reversed. Finally, we find 
Figure 4. $q$-Statistical Complexity $\mathcal{C}_{q, J}$ vs. $E_{r}$ for $10 \leq q \leq 20$. The local maximum at $E_{r}^{M}$ disappears.

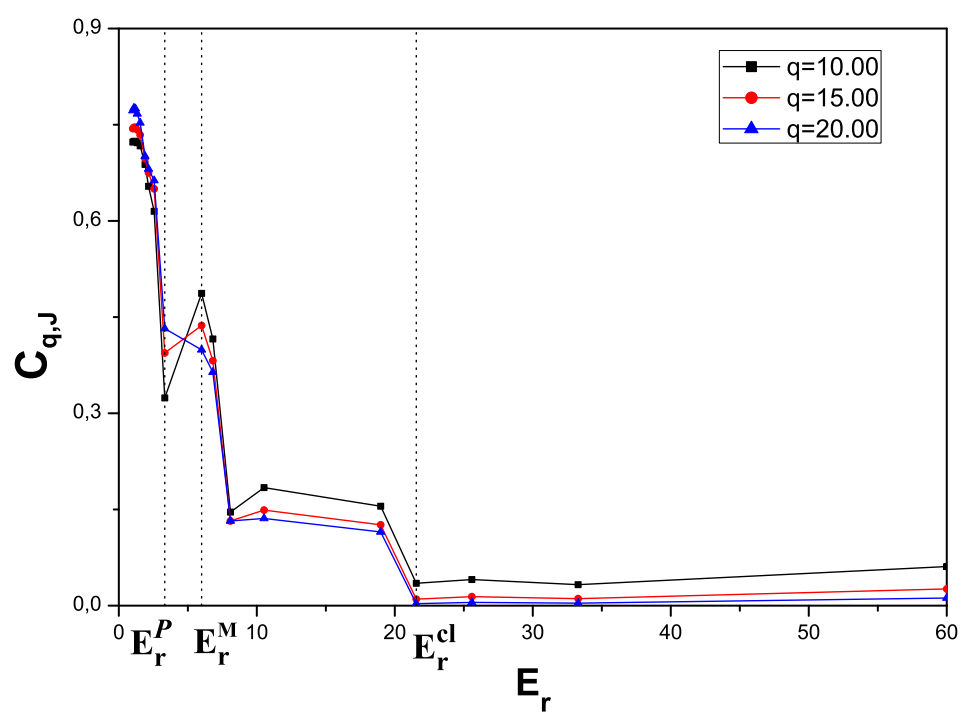

that $\mathcal{C}_{q, J}$, as a function of $q$ is a good "detector" of transitional features (see Figs. 5): a) it identifies $E_{r}{ }^{\mathcal{P}}$, starting point of the transitional sector, where chaotic behavior begins to emerge and b) it identifies $E_{r}^{M}$, i.e., it distinguishes between the two subsections into which the transitional region divides itself. These last results reconfirm a previous one obtained for $\mathcal{H}_{S_{q}}$ in [36], namely, that the parameter $q$ by itself can be regarded as the "looking glass" through which one can observe the quantum-classical transition.

\section{Acknowledgments}

AMK are supported by CIC of Argentina. The authors thank Prof. Maria Teresa Martin for her help in the computational aspects.

\section{A Normalized Tsallis wavelet entropy}

Wavelet analysis is a suitable tool for detecting and characterizing specific phenomena in time and frequency planes. The wavelet is a smooth and quickly vanishing oscillating function with good localization in both frequency and time.

A wavelet family $\psi_{a, b}(t)=|a|^{-1 / 2} \psi\left(\frac{t-b}{a}\right)$ is the set of elementary functions generated by dilations and translations of a unique admissible mother wavelet $\psi(t) . a, b \in \mathcal{R}, a \neq 0$ are the scale and translation parameters respectively, and $t$ is the time. One have a unique analytic pattern and its replications at different scales and with variable time localization.

For special election of the mother wavelet function $\psi(t)$ and for the discrete set of parameters, $a_{j}=$ $2^{-j}$ and $b_{j, k}=2^{-j} k$, with $j, k \in \mathcal{Z}$ (the set of integers) the family

$$
\psi_{j, k}(t)=2^{j / 2} \psi\left(2^{j} t-k\right) \quad j, k \in \mathcal{Z},
$$

constitutes an orthonormal basis of the Hilbert space $L^{2}(\mathcal{R})$ consisting of finite-energy signals.

The correlated decimated discrete wavelet transform provides a non-redundant representation of the signal $X$, and the values $\left\langle X, \psi_{j, k}\right\rangle$ constitute the coefficients in a wavelet series. These wavelet co- 
Figure 5. $q$-Statistical Complexity $\mathcal{C}_{q, J}$ for different $E_{r}$-values. Quantal (Figs. 5a - 5b), transitional (Figs. 5c, 5d, 5e and 5f) and classic (5g - 5h). The curves corresponding to the quantal zone resemble each other and exhibit a different aspect compared to those pertaining to the classical region.
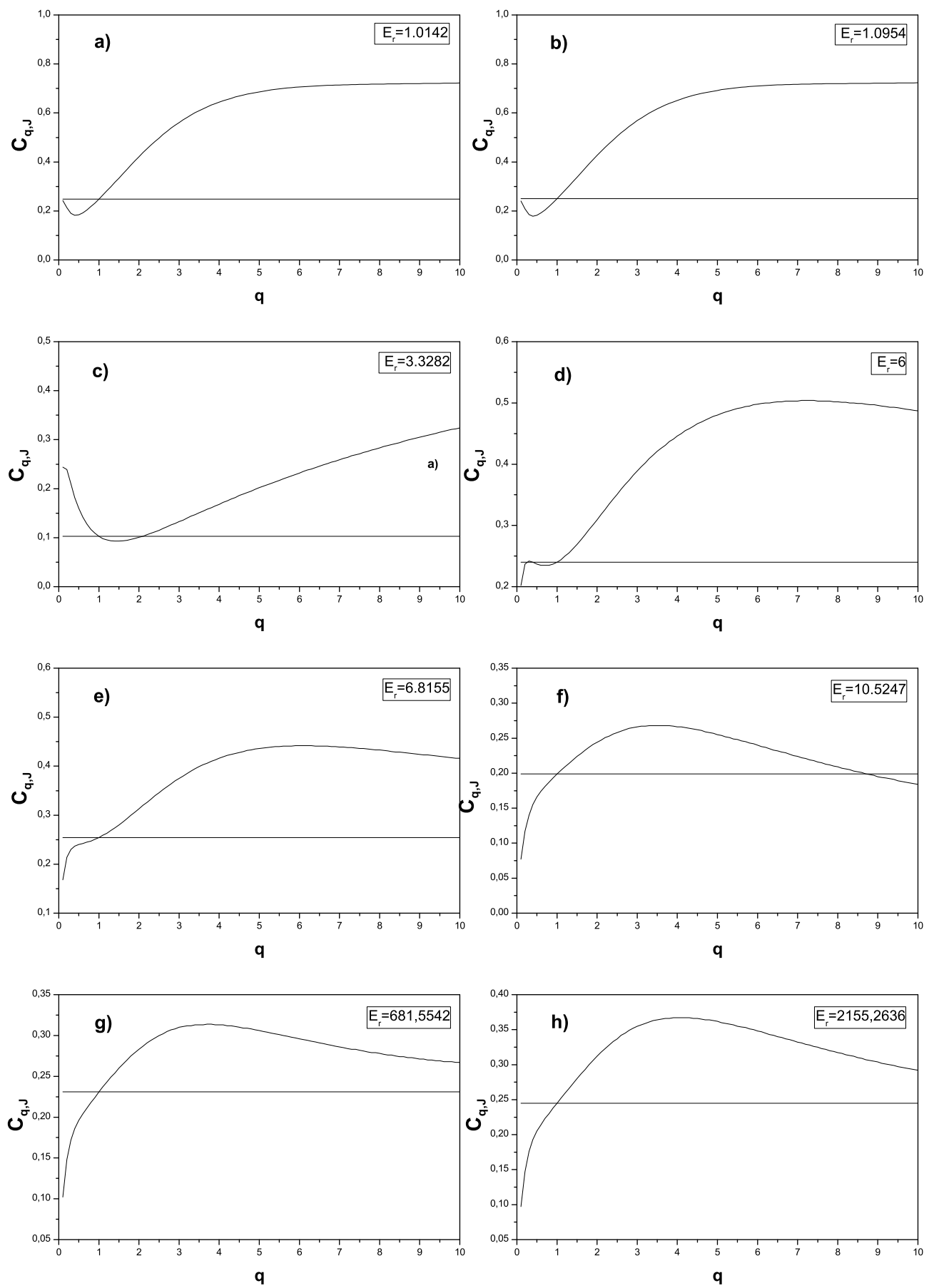
efficients provide relevant information in a simple way and a direct estimation of local energies at the different scales. Moreover, the information can be organized in a hierarchical scheme of nested subspaces called multiresolution analysis in $L^{2}(\mathcal{R})$. In the present work, we employ orthogonal cubic spline functions as mother wavelets. Among several alternatives, cubic spline functions are symmetric and combine in a suitable proportion smoothness with numerical advantages.

In what follows, the signal is assumed to be given by the sampled values $\{x(n), n=1, \cdots, N\}$. If the decomposition is carried out over all resolutions levels the wavelet expansion will read $\left(N_{J}=\log _{2}(N)\right)$

$$
X(t)=\sum_{j=-N_{J}}^{-1} \sum_{k} C_{j}(k) \psi_{j, k}(t)=\sum_{j=-N_{J}}^{-1} r_{j}(t),
$$

where $C_{j}(k)$ are the wavelet coefficients and $r_{j}(t)$ is the detail signal at scale $j$.

Since the family $\left\{\psi_{j, k}(t)\right\}$ is an orthonormal basis for $L^{2}(\mathcal{R})$, the concept of energy is linked with the usual notions derived from Fourier's theory. The signal energy, at each resolution level $j=-1, \cdots,-N_{J}$, will be the energy of the corresponding detail signal,

$$
E_{j}=\left\|r_{j}\right\|^{2}=\sum_{k}\left|C_{j}(k)\right|^{2}
$$

The total energy can be obtained in the fashion

$$
E_{t o t}=\|X\|^{2}=\sum_{j=-N_{J}}^{-1} \sum_{k}\left|C_{j}(k)\right|^{2}=\sum_{j=-N_{J}}^{-1} E_{j} .
$$

Finally, we define the normalized $p_{j}$-values, which represent the relative wavelet energy

$$
p_{j}=E_{j} / E_{t o t}
$$

for the resolution levels $j=-1,-2, \cdots,-N_{J}$. The $p_{j}$ yield, at different scales, the probability distribution for the energy. Clearly, $\sum_{j} p_{j}=1$ and the distribution $\left\{p_{j}\right\}$ can be considered as a time-scale density that constitutes a suitable tool for detecting and characterizing specific phenomena in both the time and the frequency planes.

The normalized Tsallis wavelet entropy (NTWE) is just the normalized Tsallis entropy associated to the probability distribution $P$,

$$
\mathcal{H}_{S_{q}}[P]=S_{q}[P] / S_{q, \text { max }}=\frac{1}{1-N_{J}^{1-q}} \sum_{j=-1}^{-N_{J}}\left(p_{j}-p_{j}^{q}\right),
$$

where $S_{q, \max }=\left(1-N_{J}^{1-q}\right) /(q-1)$ is attained for the equiprobable distribution $P_{e}=\left\{1 / N_{J}, \ldots, 1 / N_{J}\right\}$.

The NTWE appears as a measure of the degree of order/disorder of the time series. It provides useful information about the underlying dynamical process associated with the series. Indeed, a very ordered process can be represented by a periodic mono-frequency signal (signal with a narrow band spectrum). A wavelet representation of such a signal will be resolved at one unique wavelet resolution level, i.e., all relative wavelet energies will be (almost) zero except at the wavelet resolution level which includes the representative series frequency. For this special level the relative wavelet energy will (in our chosen 
energy units) almost equal unity. As a consequence, the NTWE will acquire a very small value. A signal generated by a totally random process or chaotic one can be taken as representative of a very disordered behavior. This kind of signal will have a wavelet representation with significant contributions coming from all frequency bands. Moreover, one could expect that all contributions will be of the same order. Consequently, the relative wavelet energy will be almost equal at all resolutions levels, and the NTWE will acquire its maximum possible value.

\section{References and Notes}

1. Shannon, C.E. A mathematical theory of communication. Bell System Technol. J. 1948, 27, 379390.

2. Shiner, J.S.; Davison, M.; Landsberg, P.T. Simple measure for complexity. Phys. Rev. E 1999, 59, $1459-1464$.

3. López-Ruiz, R.; Mancini, H.L.; Calbet, X. A statistical measure of complexity. Phys. Lett. A 1995, 209, 321-326.

4. Lamberti, P.W.; Martin, M.T.; Plastino, A.; Rosso, O.A. Instensive entropic non-triviality measure. Physica A 2004, 334, 119-131.

5. Kolmogorov, A.N.; A new metric invariant of transitive dynamic system and automorphysms in Lebesgue spaces. Dokl. Akad. Nauk SSSR 1958, 119, 861-864.

6. Sinai, Y.G.; On the concept of entropy of dynamical system. Dokl. Akad. Nauk SSSR 1959, 124, 768-771.

7. Mischaikow, K.; Mrozek, M.; Reiss, J.; Szymczak A. Construction of Symbolic Dynamics from Experimental Time Series. Phys. Rev. Lett. 1999, 82, 1144-1147.

8. Powell, G.E.; Percival, I.C. A spectral entropy method for distinguishing regular and irregular motion of hamiltonian systems. J. Phys A: Math. Gen. 1979, 12, 2053-2071.

9. Rosso, O.A.; Mairal, M.L. Characterization of time dynamical evolution of electroencephalographic records. Physica A 2002, 312, 469-504.

10. Martín, M. T.; Plastino, A.; Rosso, O. A. Statistical complexity and disequilibrium. Phys. Lett. A 2003, 311, 126-132.

11. Hanel, R.; Thurner, S. Generalized Boltzmann Factors and the Maximum Entropy Principle: Entropies for Complex Systems. Physica A 2007, 380, 109-114.

12. Tsallis, C. Possible generalization of Boltzmann-Gibbs statistics. J. Stat. Phys. 1988, 52, 479-487.

13. Alemany, P.A. ; Zanette, D.H. Fractal random walks from a variational formalism for Tsallis entropies. Phys. Rev. E 1994, 49, R956-R958.

14. Tsallis, C. Nonextensive thermostatistics and fractals. Fractals 1995, 3, 541-547.

15. Tsallis, C. Generalized entropy-based criterion for consistent testing. Phys. Rev. E 1998, 58, 1442-1445.

16. Kalimeri, M.; Papadimitriou, C.; Balasis, G.; Eftaxias, K. Dynamical complexity detection in preseismic emissions using nonadditive Tsallis entropy. Physica A 2008, 387, 1161-1172.

17. Paz, J.P.; Zurek, W.H. Quantum Limit of Decoherence: Environment Induced Superselection of Energy Eigenstates. Phys. Rev. Lett. 1999, 82, 5181-5185.

18. Emerson J.; Ballentine, L.E. Quantum-classical correspondence for the equilibrium distributions of 
two interacting spins. Phys. Rev. E 2001, 64, 026217:1-026217:11.

19. Kowalski, A.M.; Martin, M.T.; Plastino, A.; Proto, A.N. Classical Limit and Chaotic Regime in a Semi-Quantum Hamiltonian. Int. J. Bifurcation Chaos 2003, 13, 2315-2325.

20. Kowalski, A.M.; Martin, M.T.; Plastino, A.; Proto, A.N.; Rosso, O.A. Wavelet statistical complexity analysis of the classical limit. Phys. Lett. A 2003, 311, 180-191.

21. Kowalski, A.M.; Martin, M.T.; Plastino, A.; Rosso, O.A. Entropic Non-Triviality, the Classical Limit, and Geometry-Dynamics Correlations. Int. J. Mod. Phys. B 2005, 14, 2273-2285.

22. Martin, M.T.; Plastino, A.; Rosso, O.A. Generalized statistical complexity measures: geometrical and analytical properties. Physica A 2006, 369, 439-462.

23. Bloch, F. Nuclear Induction. Phys. Rev. 1946, 70, 460-474.

24. Meystre, P.; Sargent, M., III. Elements of Quantum Optics; Springer-Verlag, New York/Berlin, 1991.

25. Bulgac, A. Configurational quasidegeneracy and the liquid drop model. Phys. Rev. C 1989, 40, 1073-1076.

26. Milonni, P.W.; Shih, M.L.; Ackerhalt, J. R. Chaos in Laser-Matter Interactions; World Scientific Publishing: Singapore, 1987.

27. Kociuba, G.; Heckenberg, N. R. Controlling the complex Lorenz equations by modulation. Phys. Rev. E 2002, 66, 026205:1-026205:5.

28. Ring, P.; Schuck, P. The Nuclear Many-Body Problem; Springer-Verlag, New York/Berlin, 1980.

29. Bonilla, L. L.; Guinea, F. Collapse of the wave packet and chaos in a model with classical and quantum degrees of freedom. Phys. Rev. A 1992, 45, 7718-7728.

30. Cooper, F.; Habib, S.; Kluger, Y.; Mottola, E. Nonequilibrium dynamics of symmetry breaking in $\lambda \phi^{4}$ theory. Phys. Rev. D 199755 6471-6503.

31. Cooper, F.; Dawson, J.; Habib, S.; Ryne, R. D. Chaos in time-dependent variational approximations to quantum dynamics. Phys. Rev. E 1998, 57, 1489-1498.

32. Chung, D.J.H. Classical inflaton field induced creation of superheavy dark matter. Phys. Rev. D 2003, 67, 083514:1-083514:14.

33. Kowalski, A. M.; Martin, M. T.; Nuñez, J.; Plastino, A.; Proto, A. N. A quantitative indicator for semi-quantum chaos. Phys. Rev. A 1998, 58, 2596-2599.

34. Kowalski, A.M.; Plastino, A.; Proto, A.N. Classical limits. Phys. Lett. A 2002, 297, 162-172.

35. Kowalski, A.M.; Martin, M.T.; Plastino, A.; Proto, A.N.; Rosso, O.A. Bandt-Pompe approach to the classical-quantum transition. Physica D 2007, 233, 21-31.

36. Kowalski, A.M.; Martin, M.T.; Plastino, A.; Zunino, L. Tsallis' deformation parameter q quantifies the classical-quantum transition. [arXiv:0812.4221v1], 2008; Physica A, 2009 (in Press).

(c) 2009 by the authors; licensee Molecular Diversity Preservation International, Basel, Switzerland. This article is an open-access article distributed under the terms and conditions of the Creative Commons Attribution license (http://creativecommons.org/licenses/by/3.0/). 